\title{
In your face
}

\section{Emotional expressivity as a predictor of ideology}

Johnathan Caleb Peterson, University of Nebraska-Lincoln

Carly Jacobs, University of Nebraska-Lincoln

John Hibbing, University of Nebraska-Lincoln

Kevin Smith, University of Nebraska-Lincoln

ABSTRACT. Research suggests that people can accurately predict the political affiliations of others using only information extracted from the face. It is less clear from this research, however, what particular facial physiological processes or features communicate such information. Using a model of emotion developed in psychology that treats emotional expressivity as an individual-level trait, this article provides a theoretical account of why emotional expressivity may provide reliable signals of political orientation, and it tests the theory in four empirical studies. We find statistically significant liberal/conservative differences in self-reported emotional expressivity, in facial emotional expressivity measured physiologically, in the perceived emotional expressivity and ideology of political elites, and in an experiment that finds that more emotionally expressive faces are perceived as more liberal.

Key words: Emotion, faces, ideology

$\mathrm{P}$ eople often form judgments about others on the basis of little more than a glance. Judgments based on visual inspection of nonverbal cues - so-called thin slices of behavior - occur rapidly and reflexively and affect a broad swath of socially relevant decisions, including politically relevant judgments such as candidate evaluations, policy preferences, and vote choice. $1,2,3,4,5,6,7,8,9,10,11$ The veracity of politically relevant judgments made - quite literally - at face value is subject to a wide variety of situational factors, yet such judgments are often surprisingly accurate. ${ }^{12,13,14}$ Of particular interest here is research suggesting that people can categorize an individual's political affiliation with above-chance accuracy based solely on facial information. ${ }^{15,16,17,18,19,20,21}$ Herrmann and Shikano succinctly summarize the relevant literature thus: "These studies indicate that candidates' political inclinations can be predicted above chance level from facial images," 22 and they conclude

doi: $10.1017 /$ pls.2017.13

Correspondence: Kevin Smith, Political Science, University of Nebraska-Lincoln, Lincoln, Nebraska, United States, 68588.

Email:ksmith1@unl.edu from their own empirical analysis that "prior research is likely to be correct in asserting that ideology can be predicted above chance from facial photographs. ${ }^{23}$ This effect appears to be cross-national, ${ }^{24}$ and whatever politically relevant information is taken from faces seems to be overweighted in evaluating political characteristics. People are more likely to rely on appearance cues from faces in making judgments of political affiliation even when they have prior information that could improve the accuracy of such judgments. ${ }^{25}$

Though a considerable research literature suggests that people use faces to evaluate political traits as well as a wide range of other social traits, the specific cues driving these evaluations are far from fully understood. ${ }^{26,27,28}$ How does the face transmit information about a complex social trait such as political affiliation or orientation? Thus far, the most promising research offering an answer to this puzzle is anchored in research undertaken by Alexander Todorov and colleagues. Their research suggests that faces are perceived along two socially relevant dimensions - dominance and trustworthiness - and may provide cues on political orientation. ${ }^{29,30,31}$ Yet other facial traits are also 


\section{Peterson et al.}

argued to communicate politically relevant information, including competence, attractiveness, sociability, intelligence, and social class. ${ }^{32,33,34,35,36,37}$ Other research argues that faces simply transmit membership in broad social classifications such as age, gender, and ethnicity. As these classifications can correlate with political affiliation, people may be able to draw rapid inferences about political traits with a reasonable degree of accuracy even if there is no such thing as uniquely "liberal" or "conservative" facial cues. ${ }^{38,39}$

As there is no settled answer as to how - or even whether - individuals extract political traits from faces, it is important to investigate "the specific visual cues that people use when they draw inferences" and assess their diagnostic validity. ${ }^{40}$ This article takes up that challenge by arguing that one such diagnostic cue is emotional expressivity, which is a surprisingly little-studied candidate for cuing political information. There are good theoretical reasons to expect faces to discriminate political ideologies on the basis of emotional expressivity. Though support for these expectations does not necessarily contradict any of the hypotheses mentioned earlier - faces may transmit multiple cues for inferring political judgments - in the studies reported here, the emotional expressivity hypothesis receives empirical support even when accounting for some of these other hypotheses.

\section{Wearing your heart on your face}

The central hypothesis of this article is that, as Samochoweic, Wanke, and Fiedler put it, people who "carry their heart on their face" 41 are more likely to be tagged as liberal, while those with less emotionally expressive faces are more likely to be tagged as conservative. While there is, to the best of our knowledge, no research investigating whether politically relevant information can be conveyed by facial emotional expressivity, there is little doubt that humans employ the face and facial expressions to convey socially relevant information or that such expressions evolved to aid rapid social communication. ${ }^{42}$ Facial expressions universally communicate a fairly wide range of psychological states, especially in terms of signaling information about primary emotions such as joy, anger, disgust, and surprise. ${ }^{43}$ Facial expressions nonconsciously "leak" this emotional information to observers, though there is considerable individual-level variation in the size of the leaks. This individual-level variation in facial expressivity is stable across time within individuals - that is, it can be considered an individual-level trait, and variation in facial expressions influences evaluative judgments of social traits or classifications. ${ }^{44,45}$

Based on this research literature, we assume that faces can reliably signal emotional expressivity and that there is significant individual-level variation in facial emotional expressivity. While this assumption is noncontroversial, the primary focus of this article is whether those individual-levels differences in emotional expressivity can cue socially relevant traits and/or group affiliations such as political orientation. The key theoretical question, then, is why would nonverbal emotional expressivity encode cues to political orientation?

The theoretical frameworks employed by existing research already provide an indirect answer to this question. A good deal of the extant literature (which is often anchored in evolutionary theory) suggests that conservatives prefer more dominant-looking masculine faces and that group preferences for facial traits in leaders shift based on context, specifically preferring leaders with more masculine faces in times of threat and more feminine (or "baby-faced") features in times of peace. ${ }^{46,47,48}$ While never directly tested, these morphological features already associated with ideological and political differences have fairly direct implications for differences in expressivity. Notably, existing studies provide persuasive evidence that males tend to be less emotionally expressive than females and that males with neutral expressions - that is, an absence of affect tend to be perceived as more socially dominant. ${ }^{49,50}$ In one sense, then, the hypothesis that emotional expressivity could signal politically relevant information is simply a dynamic behavioral extension of the theoretical arguments anchored in evolutionary psychology that are already applied to facial morphology.

A more explicit theoretic framework is drawn from the work on emotional expressivity pioneered by Gross and Oliver. ${ }^{51,52,53}$ Gross and Oliver use a model of emotion that conceptualizes emotionally expressive behavior as a product of a stimulus input that triggers "emotion programs," which, in turn, drive a variety of response tendencies. Emotion programs are simply emotions - anger, happiness, and so on - that prepare an organism for action in response to a stimulus input. They do this by activating a set of response tendencies that include subjective feelings, physiological changes, and behavioral predispositions. In this model, then, variation in expressive behavior is driven by how deeply an emotion is felt and the extent to which 


\section{Emotional expressivity as a predictor of ideology}

response tendencies are activated and whether they amplify or suppress a behavioral signal of the operating emotion program. Gross and Oliver argue that the output of this model - that is, emotionally expressive behavior - is an individual-level trait that is partially innate but also shaped by social and cultural conditioning. How deeply an emotion is felt (the emotion program), and what subjective feelings, physiological responses, or behavioral reactions that emotion triggers (the response tendencies), is a product of both intrinsic predispositions and environmental influences. For example, the model explains that gender differences in emotional expressivity may be at least partially driven by how boys and girls are socialized, conditioning how deeply/shallowly emotions are felt and how response tendencies suppress/amplify those emotions.

This is important because existing research already suggests left-right political differences on the influence and expression of emotion. Conservativism as an ideology has been tied to affect avoidance, liberals have been found to be more influenced by emotion, those on the political right have been found to be higher in self-control and need for order, while those on the left are more likely to be emotionally responsive. ${ }^{54,55,56,57}$ In the context of the Gross and Oliver model, this suggests that, compared with conservatives, liberals are more likely to have response tendencies geared toward expressing rather than suppressing emotions. As ideology is increasingly seen as a product of innate predispositions and environmental conditioning, ${ }^{58}$ political differences in emotional expressivity could thus be a product of underlying innate predispositions, socialization, or, more likely, some combination of both. Regardless, within the context of the Gross and Oliver model, it is reasonable to hypothesize that response tendencies systematically vary by political orientation. If that is the case, we would predict that the model output, emotional expressivity, should also covary with political orientation.

An obvious behavioral component of emotional expressivity is facial expressivity - in other words, these individual-level differences in emotional expression should "leak" through to the face. While this serves as a reasonable theoretical basis for explaining how political information can be extracted from facial cues, to date, we are aware of no study that has actually empirically tested this hypothesis. The following four studies address the question of whether emotional expressivity can systematically cue political orientation in two different ways. The first two studies test whether liberals self-report being more emotionally expressive and whether this greater emotionally expressivity can be detected with an observational measure of facial expression. The second two studies examine whether perceptions of an individual's emotionally expressivity systematically predict perceived and actual political orientation and, if so, whether that relationship holds up when controlling for other variables - specifically, age, gender, ethnicity, dominance and trust - at the heart of alternative hypotheses on facial traits on political affiliation.

\section{Study 1}

The first study seeks to replicate and extend the findings of Gross and Oliver, ${ }^{59,60}$ who correlate self-reports of emotional expressivity with political affiliation. They do this using the Berkeley Expressivity Questionnaire (BEQ), a 16-item battery designed to tap individuallevel variation in observable emotional expression, which specifically includes facial expressions. Though the BEQ has been validated as a measure of general expressivity, Gross and Oliver ${ }^{61,62}$ emphasize that the variance captured by the BEQ falls along three distinct, orthogonal dimensions: (1) impulse strength, which captures the ability to physically control emotional expression; (2) negative expressivity, which captures the expression of negative items such as anger and fear; and (3) positive expressivity, which captures the expression of positive emotions such as happiness. We would expect liberals to score higher on all dimensions of the BEQ, but especially on impulse strength. This is because impulse strength most directly captures the concept of expressivity as a product of response tendencies acting on emotional programs (example questions: "I have strong emotions," "I am sometimes unable to hide my feelings, even though I would like to").

\section{Method}

Participants. Participants in this study were obtained through Amazon's Mechanical Turk (MTurk). Each participant received $\$ 0.50$ for their participation in this project. In all, 552 participants were recruited through MTurk, and after removing participants who did not fully complete the survey or did not answer a simple attention question, 456 participants remained. Participants ranged in age from 19 to 75 years old 


\section{Peterson et al.}

$(M=36.97, S D=12.595) .{ }^{*}$ Ideologically, $52.6 \%$ of participants reported being at least slightly liberal as opposed to the $23.2 \%$ of the sample reporting to be at least slightly conservative.

Procedures and design. After being recruited through MTurk, participants were provided a link to an online survey. They first watched a series of videos (described in more detail in Study 3), and they then completed the BEQ instrument and a 7-point self-report ideology scale ( 1 = extremely liberal, $7=$ extremely conservative), which represent measures of the key independent and dependent variables. Subjects also completed a basic demographic battery. In addition to using a simple additive scale of total expressivity taken from all BEQ items, these responses were subjected to the same analytic approach used by Gross and Oliver ${ }^{63}$ - a principal components analysis with a varimax rotation - and we found that the variance fell into the same three-factor impulse strength/positive expressivity/negative expressivity structure with very similar factor loadings for individual items. This result provides confidence in the psychometric properties of the BEQ in the sample, and the factor scores from each of the three dimensions as independent variables are used in the following analyses.

\section{Results}

Table 1 reports the simple correlations between all expressivity measures, a 7-point ideology score ( $1=$ very liberal, $7=$ very conservative $)$ and a measure of partisan self-identification $(1=$ Democrat, $2=$ independent, 3 = Republican). The latter was included not only to track pattern similarities in ideology and partisanship but also to allow direct comparison of results with those of Gross and Oliver, who examined partisanship but not ideology. Our theoretical framework predicts that all correlations should be negative. As reported in Table 1, six of eight correlations conform to those expectations, with four of the six statistically significant at $p<0.05$, and one at $p<0.10$.

In terms of partisanship, total expressivity $(r=$ $-0.10, p<0.05)$ and impulse strength $(r=-0.11, p<$

\footnotetext{
* We expected effect sizes in all studies to be modest. The sample size required to detect effects of $r=0.15$ with $80 \%$ power is approximately 350 (sample size required for $r=0.15, \alpha=0.05$, and $\beta=0.20$ is $N=347)$. On that basis, the samples in Study 1 and Study 3 $(N=456)$, as well as Study $4(N=795)$, have more than sufficient power. Study 2, however, is underpowered $(N=227)$, though the statistically significant results tend to have slightly higher effect sizes (mean $r=0.16$ ).
}

Table 1. Self-reported emotional expressivity, ideology and partisanship.

\begin{tabular}{lcc}
\hline \hline Emotional dimension & Ideology & Partisanship \\
\hline Total expressivity & -0.06 & $-0.10^{*}$ \\
Impulse strength & $-0.14^{*}$ & $-0.10^{*}$ \\
Positive expressivity & $0.10^{*}$ & 0.01 \\
Negative expressivity & -0.04 & $-0.09 \#$ \\
\hline${ }^{*} p<0.05 ; \# p<0.10$. & &
\end{tabular}

$0.05)$ are significant and negatively related to being a Republican; negative expressivity $(r=-0.05, p=0.29)$ and positive expressivity $(r=0.01, p=0.90)$ show no relationship with partisanship. The results for ideology are mixed. The strongest relationship is for impulse strength and ideology $(r=-0.14, p<0.01)$. This relationship clearly conforms to our expectations - when it comes to strong emotional reactions accompanied by physical and behavioral changes difficult to stop or hide, conservatives report less and liberals report more. Although negative expressivity $(r=-0.03, p=0.43)$ and total expressivity $(r=-0.06, p=0.18)$ are in the expected direction, neither measure is statistically significant. However, positive expressivity $(r=0.09$, $p<0.05)$ is significantly correlated with ideology and the correlation is positive, which is in the opposite direction hypothesized. Other studies find conservatives report being happier and more optimistic than liberals. So, perhaps it makes sense this generally sunnier outlook on life might be reflected in the expression of positive emotions. ${ }^{64,65}$

The key finding in support of the hypotheses, then, is impulse strength: compared with liberals, conservatives are much less likely to report having strong emotional reactions, typically accompanied by physical and behavioral changes such as frowning and smiling. ${ }^{66}$ Table 2 reports the results of a regression analysis designed to examine how robust this finding is. The model specification is fairly straightforward, containing a series of known correlates of ideology: age (in years), gender $(1=$ male), income (a 10-point scale moving in $\$ 10,000$ increments from $1=\$ 20,000$ or less to $10=\$ 100,000$ or more), religious attendance (a 5-point scale where $5=$ attend religious services more than once and $1=$ less than once a year), and race $(1=$ white, $0=$ other $)$. To this basic specification the BEQ measures were added, one model with total expressivity and one model with the three individual, orthogonal components of emotional expression. 


\section{Emotional expressivity as a predictor of ideology}

Table 2. Ideology and emotional expressivity.

\begin{tabular}{|c|c|c|}
\hline Variable & $\begin{array}{l}\text { Ideology } \\
\text { (Model 1) }\end{array}$ & $\begin{array}{l}\text { Ideology } \\
\text { (Model 2) }\end{array}$ \\
\hline Age & $\begin{array}{c}0.00 \\
(0.01)\end{array}$ & $\begin{array}{c}0.00 \\
(0.00)\end{array}$ \\
\hline Male & $\begin{array}{c}0.40^{*} \\
(15)\end{array}$ & $\begin{array}{l}0.32^{*} \\
(0.15)\end{array}$ \\
\hline Income & $\begin{array}{l}0.06^{*} \\
(0.03)\end{array}$ & $\begin{array}{l}0.05^{*} \\
(0.02)\end{array}$ \\
\hline Religious attendance & $\begin{array}{l}0.42 * \\
(0.42)\end{array}$ & $\begin{array}{l}0.41 * \\
(0.06)\end{array}$ \\
\hline White & $\begin{array}{l}0.37^{*} \\
(0.18)\end{array}$ & $\begin{array}{l}0.39 * \\
(0.18)\end{array}$ \\
\hline Total expressivity score & $\begin{array}{l}-0.00 \\
(0.00)\end{array}$ & - \\
\hline Impulse strength & - & $\begin{array}{c}-0.17^{*} \\
(0.07)\end{array}$ \\
\hline Positive expressivity & - & $\begin{array}{c}0.11 \\
(0.07)\end{array}$ \\
\hline Negative expressivity & - & $\begin{array}{l}-0.05 \\
(0.07)\end{array}$ \\
\hline Constant & $\begin{array}{l}2.23^{*} \\
(0.46)\end{array}$ & $\begin{array}{l}2.02^{*} \\
(0.29)\end{array}$ \\
\hline$N$ & 457 & 457 \\
\hline$F$ & $11.56^{*}$ & $9.73^{*}$ \\
\hline Adjusted $R^{2}$ & 0.12 & 0.13 \\
\hline \multicolumn{3}{|c|}{$\begin{array}{l}\text { Note: The dependent variable is a } 7 \text {-point Likert scale measuring } \\
\text { ideology where } 1=\text { strongly liberal, } 4=\text { moderate, middle of the road, } \\
\text { and } 7=\text { strongly conservative. Male and white are dummy coded } \\
\text { where } 1=\text { male and white, respectively. }{ }^{*} p<0.05 \text {, unstandardized } \\
\text { coefficients (standard errors) reported. }\end{array}$} \\
\hline
\end{tabular}

The results of this analysis, reported in Table 2, reinforce the key inference. In terms of self-reported emotional expressivity, what systematically differentiates between conservatives and liberals is impulse strength. The standardized betas for Model 2 indicate that impulse strength $(\beta=-0.11, p<0.05)$ has a slightly bigger impact on ideological self-report than gender $(\beta=-0.10, p<0.05)$, income $(\beta=0.09$, $p<0.05)$, and race $(\beta=0.10, p<0.05)$; only religious attendance had a bigger standardized beta $(\beta=0.30$, $p<0.05)$. The latter is not unexpected as religious attendance is a robust predictor of ideology; for example, in the U.S. population, approximately $55 \%$ of self-identified conservatives report attending religious services weekly, compared with $27 \%$ of liberals. ${ }^{67}$

\section{Discussion}

This initial study, then, provides evidence that liberals and conservatives (and Democrats and Repub- licans) self-report different types and levels of emotional expressivity. Specifically, liberals are more likely to have higher impulse strength scores - that is, they report having a harder time controlling the expression of strong emotions. Given the specific purpose of the BEQ, those displays of emotion are clearly expected to be expressed physically and behaviorally, which implies they will be expressed facially.

\section{Study 2}

Although supporting the key hypothesis, the results of Study 1 are reliant on self-reports. Study 2 empirically tests this hypothesis with an observational behavioral measure, the degree of facial emotional expressivity in individuals, measured using facial electromyography (EMG). Specifically, EMG measures of the corrugator supercilii were captured. We chose this muscle for measurement of facial expressivity for several reasons. First, of the various muscles and muscle groups responsible for facial expressions, the corrugator tends to give the clearest signal. Its physiological characteristics - bilateral innervations, limited representation in the motor cortex, and an absence of overlapping muscle groups - mean that it is not subject to fine voluntary motor control or susceptible to disruption by the activation of other muscles. Additionally, the corrugator tends to activate in a linear and reciprocal fashion based on the valence of the stimulus content. In other words, negative images increase corrugator reactivity, while positive images decrease reactivity. ${ }^{68}$

The corrugator is also attractive as a test of our theoretical argument because it is centrally involved in creating facial expressions associated with impulse control. BEQ items that discriminate impulse control include "I sometimes cry during sad movies," "My body reacts very strongly to emotional situations," "I experience my emotions very strongly," and "There have been times when I have not been able to stop crying even though I tried to stop." The physical manifestation of such emotional experiences clearly implies corrugator activation - that is, a muscle that is involuntarily activated or deactivated in response to felt emotions.

As the corrugator is frequently associated with the display of negative primary emotions (e.g., disgust, anger, fear), it also makes an interesting target for investigation in light of a range of studies that suggest that conservatives have a more pronounced negativity bias than liberals. ${ }^{69}$ This would seem to infer the hypothesis that conservatives rather than liberals would 


\section{Peterson et al.}

show the greater corrugator activation, at least in response to negatively valenced images. The case for hypothesizing the opposite - that liberals will show greater corrugator activity across a range of differentially valenced stimuli - rests on several threads. The most important, of course, is the theoretical case made earlier. If liberals are indeed more emotionally expressive, and the face "leaks" emotion, it makes sense that the facial muscles involved in signaling primary emotions would be active for liberals. That case is also backed by a range of findings in the literature.

The primary physiological evidence of a conservative negativity bias is electrodermal activity (EDA), which is widely used and accepted as a measure of sympathetic nervous system arousal. This does not necessarily imply a similar relationship for electromyographic measures. EDA and facial EMG tap largely independent dimensions of emotion (respectively, arousal and valence), and these measures are not typically correlated. It is also notable that emotional suppression - keeping a neutral expression while viewing emotionally evocative stimuli - decreases EMG activity and increases EDA. ${ }^{70,71,72,73}$ That pattern is perfectly consistent with the notion of increased facial EMG for liberals and increased SCL responses for conservatives.

\section{Method}

Participants. A professional survey organization recruited a random sample of 342 adults (184 female) between the ages of 19 and $65(M=45.6, S D=12.94)$ from a midsized midwestern city that is home to a large research university. Participants were paid $\$ 50$ for their time. Moderates $(n=115)$ were removed from the analysis, as the concern here is centered on whether facial expressivity can tag people with clear political leanings or affiliation, not the absence of those leanings or affiliations. After moderates were removed, liberals $(14.6 \%)$ and liberal leaners $(28.4 \%)$ made up $43 \%$ of the remaining sample, while conservatives $(25.3 \%)$ and conservative leaners $(31.5 \%)$ made up the rest.

Procedures and design. Participants proceeded to a physiology lab, where sensors were attached to record a set of physiological variables including heart rate, rate of respiration, electrodermal response, and electromyographic activity across several facial muscles. Corrugator activation was measured using 5-millimeter surface electrodes filled with a conductive gel. Prior to the application of the electrodes, the skin was cleaned and debraded with an exfoliating gel and isopropyl alcohol. ${ }^{74}$
The pair of surface electrodes measuring corrugator activity was placed just above the left eyebrow, with the positive electrode aligned with the inside corner of the eye and the negative electrode immediately next to it above the middle of the eye. A separate electrode was placed behind the left ear as a ground to reduce noise in the signal.

After all the sensors were in place, participants viewed a series of randomly ordered still images shown on-screen for 10 seconds each. Images were separated by an interstimulus interval of similar length consisting of a black screen with a fixation point (a small white cross). The images used in this analysis were taken from the International Affective Picture System, a data set of images prevalidated for their valence and arousal. ${ }^{75}$ These images were also independently rated for valence (scale: 1 = positive to 9 = negative), arousal, and content by participants at the end of the lab session. The subjects unambiguously rated four of these images as emotionally neutral (e.g., an iron and a dustpan), eight as disgusting (e.g., vomit and roaches on food), four as threatening (e.g., a pointing gun and an assailant putting a knife to a victim's throat), and four as positive (e.g., a happy baby and a spectacular waterfall).

EMG activity (in microvolts, $\mu \mathrm{V}$ ) was recorded over the course of the experimental protocol and averaged over time for each individual image and inter-stimulus interval. As is often the case, all the data exhibited a significant positive skew and required an adjustment using a square-root transformation. ${ }^{76}$ The results reported here are based on the square root of the electrical activity of the corrugator muscle. Reactivity for each individual image was calculated by subtracting the average corrugator activity recorded during the interstimulus interval occurring immediately prior to the image of interest from the average corrugator activity during the image itself.

\section{Results}

The key test comes in the analysis of all images and the threatening, disgusting, and positive images. Response to neutral images is included as a reference and control. In contrast to other categories of images, because the neutral images are expected to evoke no emotional response, and thus no significant corrugator activity, EMG measures are not expected to systematically discriminate ideological leaning in response to such images.

Preliminary evidence can be seen in Figure 1. This figure shows ideological differences in mean corrugator 


\section{Emotional expressivity as a predictor of ideology}

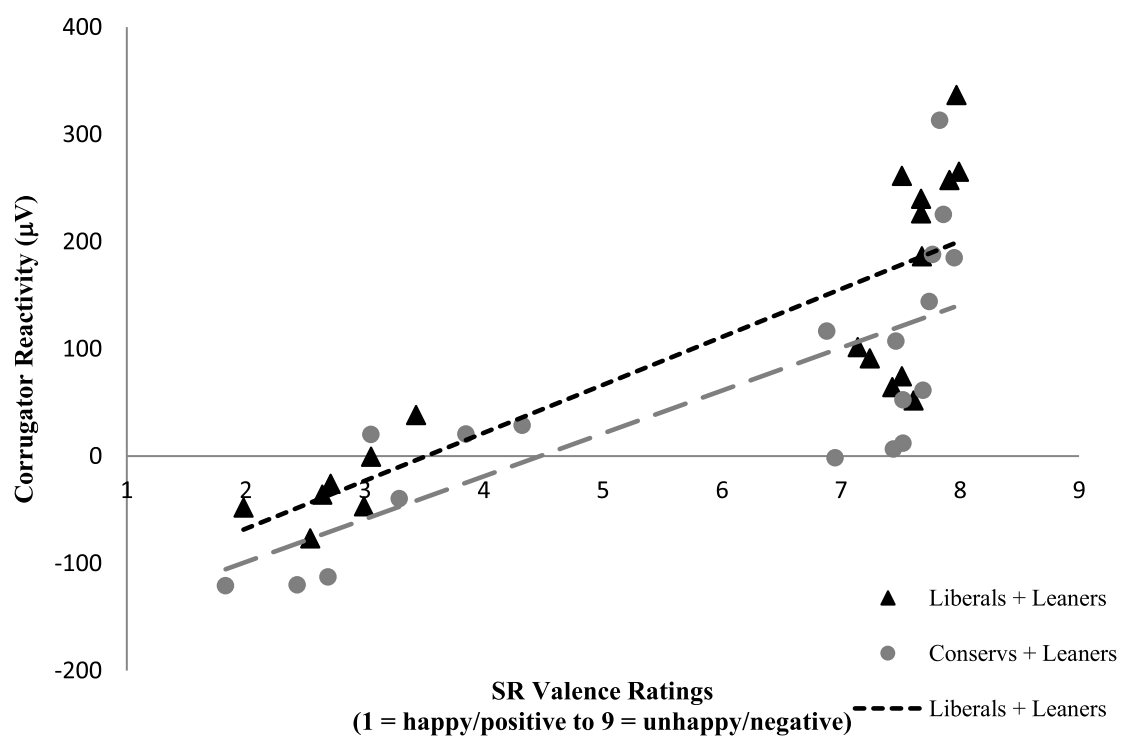

Figure 1. Comparing valence ratings and corrugator reactivity for individual images. Note: Each $\mathbf{\Delta} / \bullet$ represents a single image (e.g., happy baby, roach on food, etc.) as identified by its mean valance rating on the $x$-axis and the average reactivity across the corrugator muscle while the image was viewed by participants on the $y$-axis.

reactivity as a function of the mean self-reported emotional valence (positive to negative) for each image with an identifiable emotional valence (disgusting, threatening, and positive). Taken as a whole, this plot shows the hypothesized relationship between corrugator activity and stimulus valence, that is, positively valenced images deactivate the corrugator and negatively valenced images (e.g., those with threating and disgusting content) activate the corrugator. However, it is also clear that the relationship between self-reported emotional valence and reactivity is different for liberals (represented by triangles) and conservative (represented by circles). The slopes in Figure 1 plot the relationship between self-reported image valence and corrugator activity separately for conservatives and liberals. As can be seen, the slopes are similar (the liberal slope is actually slightly steeper), but the intercepts are different. The difference in those intercepts represents variation in mean corrugator reactivity to all images; that difference is fairly substantial (a mean difference of $52 \mu \mathrm{V}$ ) and statistically significant $(t(226)=2.15, p=0.03)$. Liberals, in short, have a consistently higher degree of corrugator activity for all images, suggesting they have more emotionally expressive faces.

Table 3 presents correlations between ideology and corrugator reactivity in response to all images and to particular categories of images. The direction of the relationship between ideology and corrugator reactivity
Table 3. Correlations and partial correlations: Ideology by EMG reactivity $(\mu \mathrm{V})$.

\begin{tabular}{lccc}
\hline \hline $\begin{array}{l}\text { Image } \\
\text { content category }\end{array}$ & No controls & $\begin{array}{c}\text { Controls: } \\
\text { gender only }\end{array}$ & $\begin{array}{c}\text { Controls: gender, } \\
\text { age, education }\end{array}$ \\
\hline Overall & $0.145^{*}$ & $0.136^{*}$ & $0.136^{*}$ \\
Threat & $0.168^{*}$ & $0.157^{*}$ & $0.150^{*}$ \\
Disgust & 0.089 & 0.079 & 0.077 \\
Positive (non-erotic) & $0.159^{*}$ & $0.179^{* *}$ & $0.196^{* *}$ \\
Neutral & 0.021 & 0.026 & 0.025 \\
\hline
\end{tabular}

Note: Ideology: conservatives $=0$, liberals $=1 .^{*} p<0.05 ;{ }^{* *} p<0.01$ $* * * p<0.001$.

in this table is perfectly consistent - all correlations are positive, indicating that liberals have higher corrugator activity across all categories of emotional stimuli. All correlations for threat, positive, and combined stimulus categories are statistically significant. Neutral images are statistically insignificant; this is an expected finding given the absence of valence in these stimuli. The only real surprise is the consistent statistical insignificance of images rated as disgusting. Though consistently in the expected direction, this coefficient never reaches conventional standards of statistical significance; even directional (one-tailed) tests put the probability levels just outside conventional levels of statistical significance (estimated probabilities are approximately 0.12). 


\section{Peterson et al.}

\section{Discussion}

The preliminary analysis in Figure 1 came with some important caveats. Critically, there was the potential for a spurious relationship. The relationship between corrugator activity and ideological self-identification may be driven by a separate, independent relationship that both of these measures share with another variable. The obvious candidate for such a role is gender. If females are more emotionally expressive and also more likely to be liberal, then perhaps Figure 1 reflects a relationship between gender and corrugator reactivity rather than a relationship between ideology and corrugator reactivity. The analysis reported in Table 3 sought to address this concern. Still, the weight of the empirical evidence in this analysis clearly is supportive of the general hypothesis: as measured by actual corrugator activity in response to a range of emotionally valenced stimuli, liberals have more expressive faces.

\section{Study 3}

The first two studies established that liberals and conservatives differ in terms of emotionally expressivity, but they did not examine whether this facial variation systematically transmits signals about political affiliation. Study 3 examines whether people associate perceived emotional expressivity with ideology while controlling for the potential confounds of age, ethnicity, and gender. The analysis in Study 3 is directed at two questions: Does the perceived emotional expressivity of congressmen systematically map onto their perceived ideology? Does the perceived emotional expressivity of congressmen improve accuracy in classification of their actual ideology?

\section{Method}

Participants. The sample is the same one utilized in Study 1. Again, participants in this study were obtained though MTurk. Each participant received $\$ 0.50$ for their participation in this project. In all, 552 participants were recruited through MTurk, and after removing participants who did not fully complete the survey or did not answer a simple attention question, 456 participants remained. Participants ranged in age from 19 to 75 years old $(M=36.97, S D=12.595)$.

Procedures and design. After being recruited into the study, participants were told they would be watching a series of short videos without sound. They were then shown 10 short videos of members of Congress who served during the 108th Congress. The 10 videos featured five of the most liberal members of Congress, as defined by their DW-NOMINATE scores, and five of the most conservative members of the 108th Congress. Care was taken to ensure that the members of Congress did not vary substantially in terms of age, sex, and race; all were older, male, white, dressed similarly, and appeared in an identical setting - a lectern on the floor of the House of Representatives with no other individuals visible. Furthermore, none of these members of Congress was particularly well known; none had key leadership positions or particularly high media profiles. In short, the videos were of five little-known, older, male, white, and liberal members of Congress and five little-known, older, male, white conservatives.

The relative obscurity of these congressmen is important, as the key variable is respondents' perceptions of the ideology of the congressmen, and we wanted to exclude any members likely to be recognized. Five-second clips were edited so they included no obvious body movements or demonstrative emotional facial expressions, such as laughter. The videos were presented without sound to avoid any vocal cues to emotional state and showed the congressmen from approximately chest height up. To the greatest extent possible, the aim in the videos was to give subjects a set of similarly looking political leaders, in an identical environment, where faces with relatively neutral expressions were the dominant visual image, and to see whether some were perceived as more emotionally expressive and/or more liberal or conservative.

The videos were randomized by participant and presented one at time. After viewing a video, respondents were asked a series of five questions pertaining to the perceived emotional expressivity, political ideology, athleticism, and marital faithfulness (all 7-point scales) of the congressman in the video along with an item asking participants if they recognized the congressman in the preceding video (on average, approximately $95 \%$ of the sample could not recognize individual congressmen).

\section{Results}

The key test of the first question - does the perceived emotional expressivity of these congressmen systematically map onto their perceived ideology - is reported in Table 4. These are partial correlations between the subjects' perceived emotional expressivity and the subjects' perceived ideology of the 10 congressmen. Subject age, gender, ideology, emotional expressivity (total 


\section{Emotional expressivity as a predictor of ideology}

Table 4. Perceived emotional expressivity and perceived ideology of congressmen.

\begin{tabular}{lc}
\hline \hline Liberals & Conservatives \\
\hline Stark: $-0.23^{*}$ & Crane: $-0.12^{*}$ \\
Miller: $-0.12^{*}$ & Duncan: $-0.16^{*}$ \\
Olver: $-0.15^{*}$ & Rohrabach: $-0.13^{*}$ \\
Hinchey: $-0.21^{*}$ & Sensenbrenner: -0.07 \\
McDermott: $-0.18^{*}$ & Shadegg: $-0.19^{*}$ \\
Mean $r-0.18$ & -0.13 \\
\hline
\end{tabular}

Note: ${ }^{*} p<0.05$, partial correlations between perceived emotional expressivity and perceived ideology, controlling for subjects age, gender, ideology, emotional expressivity, and whether they recognized the congressman.

BEQ score), and recognition of the congressmen were controlled for.

Even with the controls just listed, the correlations clearly and consistently support the hypothesis. All of the coefficients are negative, and 9 of the 10 are statistically significant. So, as perceived emotional expressivity of the congressmen increased, they were judged as more liberal. This effect also seems to be slightly larger for liberals than for conservatives. The mean correlation between perceived expressivity and ideology was 0.18 for liberals and 0.13 for conservatives. Furthermore, the mean perceived emotional expressivity for liberal congressmen was 4.01, whereas the comparable mean for conservative congressmen was $3.56(t(456)=11.4$, $p<0.001$, paired-sample $t$-test).

What of the second objective - does emotional expressivity systematically map onto more accurate evaluations of ideology? To assess this question, we created measures of the accuracy of subject evaluations of legislator ideology. For each legislator, we created a dummy variable where $1=$ subject correctly identified congressman as liberal/conservative $0=$ subject incorrectly identified congressman as liberal/conservative. For each subject, we simply summed the number of correct identifications into two separate indexes, one for liberal legislators and one for conservative legislators. This gives us two 0-5 indexes of the accuracy of subject ideological identification of the congressmen (median identified correctly was 2.0 for liberal legislators and 3.0 for conservatives, respectively, slightly below and above chance rates). We then correlated these indexes with the mean perceived emotional expressivity scores given liberal and conservative legislators. If emotional expressivity cues accurate ideological classification, we would predict a positive and significant relationship for liberals and a negative and significant relationship for conservatives. In other words, subjects who viewed liberal legislators as more emotionally expressive should be more accurate in identifying their ideology, and subjects who viewed conservative legislators as less emotionally expressive should be more accurate in identifying their ideology. The actual correlation for liberal congressmen was $r=0.13(p=0.00)$, and for conservative congressmen it was $r=-0.08(p=0.07)$. This suggests that higher evaluations of emotional expressivity improved the accuracy of ideological prediction for liberal congressmen. The results for conservatives are in the expected direction, i.e. lower evaluations of emotional expressivity correlated with more accurate classifications, but as the result is just outside traditional levels of statistical significance, the evidence here is somewhat weaker. The basic direction and effect sizes hold even using a variety of control (the same variables used for the partial correlations used in Table 4).

\section{Discussion}

Overall, then, the results of Study 3 suggest perceived emotional expressivity does correlate with perceived political orientation and with actual political orientation. The evidence is stronger for the former than the latter finding, but the findings thus far offer a fairly clear response to this study's first objective. Even though the vast majority (approximately $95 \%$ ) reported having no idea who they were watching, subjects in the sample clearly evaluated liberal elites to be more emotionally expressive, and this perceived emotional expressivity consistently predicts evaluations of ideology. Perceived emotional expressivity also seems to correlate with actual political orientations.

\section{Study 4}

The final study assesses whether evaluations of political orientation differed for the same individual based on whether they displayed a neutral or an emotionally demonstrative facial expression.

\section{Method}

Participants. Participants comprised an MTurk sample of 795 subjects reasonably similar sociodemographically to the MTurk sample used in Study 1 and to MTurk samples generally (age $M=31.8, S D=11 ; 74 \%$ white; $58 \%$ reporting at least slightly liberal).

Procedure and design. After agreeing to participate, subjects were told they were about to view an image 


\section{Peterson et al.}

for one second and then asked a series of questions about that image. They were then randomly assigned to view one of four images. Two of images consisted of a white, middle-aged, German male liberal politician from the European Parliament, and two consisted of a white, middle-aged, German male conservative politician in the European Parliament. In one image the liberal/conservative was shown with a neutral expression and in the other with a clear emotional expression (smiling), thus giving the four target images: a liberal with a neutral expression, a liberal with a clear emotional expression, a conservative with a neutral expression, and a conservative with a clear emotional expression. The politicians were chosen by examining the NOMINATE scores of members in the European Parliament as calculated by Hix, Noury, and Roland. ${ }^{77}$ After limiting the search to German members of the Fifth European Parliament (1999-2004), the male politicians furthest to the left and furthest to the right for whom emotionally expressive and neutrally expressive images could be found on the European Parliament's website were chosen. The images were then converted into black and white, and facial hair (a mustache for the conservative politician) and eyeglasses (both politicians) were removed using Photoshop. The target images can be seen in Figure 2; it should be noted these images were selected to hold age, gender, and ethnicity cues constant.

After being exposed to the target image, subjects were asked the degree to which the person in the image displayed 19 traits. The response categories were on a 5 -point, where $1=$ strongly disagree and $5=$ strongly agree. These included subject ratings on the degree to which the target image was seen as emotionally expressive, politically liberal, and a series of traits designed to replicate the dominance/trustworthy facial dimensions reported in a series of studies by Todorov and colleagues. ${ }^{78}$ Subjects were also asked a series of demographic questions.

\section{Results}

The analyses are presented in Table 5 . Model 1 is a $2 \times 2$ factorial ANOVA reported in a regression format. In Model 1, there are dummy variables indicating whether the randomly assigned target image is liberal $(1=$ liberal, $0=$ conservative $)$ and expressive $(1=\mathrm{ex}-$ pressive, $0=$ neutral), as well as an interaction between these two variables. The intercept, then, is the mean evaluation of how politically liberal the target image is in the neutral, conservative condition - that is, when both the dummy variables and the interaction equal
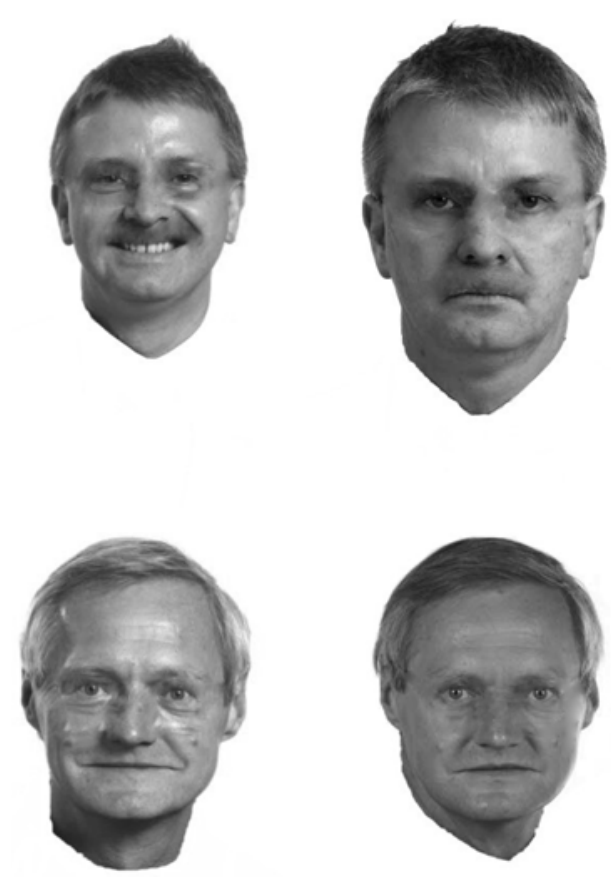

Figure 2. Images used for experimental manipulation. Note: The right-wing politician is on the top, and the left-wing politician is on the bottom. The images on the left are emotionally expressive, and the images on the right are neutrally expressive.

zero. Given the coding scheme, positive coefficients for the condition dummies indicate subjects in those conditions are, on average, evaluating the target image as more liberal. As can be seen, subjects in the liberal condition did indeed evaluate the target image as more liberal than subjects in the conservative condition, and subjects in the expressive condition evaluated the target image as more liberal than subjects in the neutral condition. The differences are fairly modest - a 0.33-point difference $(p<0.05)$ in the ideological condition and a 0.53 -point difference $(p<0.05)$ in the emotional condition - but they are statistically significant and in the expected direction. The interaction is not in the expected direction, but the coefficient is small and not statistically significant.

These results suggest the liberal target is perceived as more liberal and the emotionally expressive targets are perceived as more liberal, but also, these are independent effects. As the conservative or liberal target shifts from neutral to emotionally expressive, evaluations of that target shift reliably to the political left. The mean evaluation of political orientation of the nonexpressive 


\section{Emotional expressivity as a predictor of ideology}

Table 5. Evaluations of political orientation and emotional expressivity.

\begin{tabular}{|c|c|c|c|c|}
\hline Variable & Model 1 & Model 2 & Model 3 & Model 4 \\
\hline Intercept & $\begin{array}{l}2.29^{*} \\
(0.07)\end{array}$ & $\begin{array}{l}2.08^{*} \\
(0.10)\end{array}$ & $\begin{array}{l}1.57^{*} \\
(0.28)\end{array}$ & $\begin{array}{l}1.35^{*} \\
(2.9)\end{array}$ \\
\hline Liberal condition & $\begin{array}{l}0.332^{*} \\
(0.09)\end{array}$ & & & \\
\hline Emotion condition & $\begin{array}{l}0.52^{*} \\
(0.53)\end{array}$ & & & \\
\hline $\begin{array}{l}\text { Liberal } x \\
\quad \text { Emotion interaction }\end{array}$ & $\begin{array}{l}-0.17 \\
(0.13)\end{array}$ & & & \\
\hline Expressivity evaluation & & $\begin{array}{l}0.19^{*} \\
(0.03)\end{array}$ & & $\begin{array}{l}0.08^{*} \\
(0.03)\end{array}$ \\
\hline Dominance & & & $\begin{array}{l}-0.01 \\
(0.00)\end{array}$ & $\begin{array}{l}-0.01 \\
(0.00)\end{array}$ \\
\hline Trust & & & $\begin{array}{l}0.06^{*} \\
(0.01)\end{array}$ & $\begin{array}{l}0.06^{*} \\
(0.01)\end{array}$ \\
\hline$N$ & 794 & 794 & 794 & 794 \\
\hline $\boldsymbol{F}$ & $20.87^{*}$ & $36.07^{*}$ & $59.17^{*}$ & $42.8^{*}$ \\
\hline Adjusted $\boldsymbol{R}^{2}$ & 0.07 & 0.05 & 0.13 & 0.14 \\
\hline
\end{tabular}

Note: For the dependent variable, respondents were asked to agree or disagree that the trait "politically liberal" described the person they saw. Higher values for the DV indicate the respondent agreed that the trait "politically liberal" described the person they saw. Liberal condition $=1$ if the left-wing politician was shown. Emotion condition $=1$ if an emotionally expressive politician was shown. ${ }^{*} p<0.05$, unstandardized coefficients (standard errors) reported.

liberal target was 2.62 on the 5 -point scale and 2.98 for the expressive liberal, a modest (0.36-point) but statistically significant difference $(t=3.65, p<0.001)$. The shift from neutral to expressive was larger for the conservative target, mean evaluations moving from 2.29 to 2.82 , or a difference of 0.52 points between conditions $(t=6.11, p<0.001)$.

Model 2 in Table 5 is a simple bivariate regression predicting evaluations of political orientation using only evaluations of emotional expressivity $(1=$ strongly disagree target is emotionally expressive, $5=$ strongly agree). This process establishes a baseline empirical finding that perceived emotional expressivity overall does indeed systematically covary with perceived ideology, and the significant positive coefficient confirms this.

Model 3 in Table 5 regresses evaluations of ideology on indices of trustworthiness and dominance suggested by Oosterhof and Todorov. ${ }^{79}$ There is a somewhat mixed result here. The coefficient for the trustworthiness index is positive and statistically significant, meeting the expectation more trustworthy faces will be evaluated as more liberal. The coefficient for the dominance index is in the expected direction - that is, negative meaning more dominant faces are viewed as more conservative, but not statistically significant. The level of significance associated with this coefficient, however, is fairly close to standard thresholds $(p=0.072)$; though caveats clearly apply, this result as inconsistent with a priori expectations.

Model 5 combines Models 2 and 3, predicting perceived ideology using perceived emotional expressivity, trust, and dominance. In this model, emotional expressivity and trust continue to demonstrate statistically significant effects, but dominance does not (though it continues to be in the expected direction). This fact is taken as evidence the degree to which a face is perceived to be emotionally expressive influences evaluations of political orientation independent of the two facial traits widely demonstrated to have social relevance in the existing literature.

\section{Discussion}

These findings suggest that emotionally expressivity influences evaluations of political orientation, with more expressive faces - regardless of whether they belong to a liberal or a conservative - pushing evaluations marginally to the political left. The significant, independent effect of the ideology condition, however, also clearly suggests something else about faces transmitting ideological cues. Moreover, whatever that something is, it is not likely to be age, gender, or ethnicity given the selection of the target images. To establish what that something else might be, the impact of overall evaluations of emotional expression in conjunction with judgments on the dominance/trust facial dimensions suggested by Oosterhof and Todorov ${ }^{80}$ was investigated. It was found that expressivity worked independently of these dimensions, and dominance did not play a role.

A caveat that should be noted for this study is that we only examined a single, positively valenced emotional expression (i.e., a smile). So while our findings fit with our general theoretical expectations, appropriate caution should be exercised in generalizing to emotional expressivity more generally.

\section{Conclusion}

The empirical results from the studies reported here support four key inferences: (1) liberals and conservatives self-report different types and levels of emotional expressivity, with the key differences centering 


\section{Peterson et al.}

on what Gross and Oliver label impulse strength ${ }^{81}$; (2) an objective measure of facial emotional expressivity consistently discriminates between liberals and conservatives; (3) political leaders perceived as more emotionally expressive are more likely to be judged liberal, and those judgments of political orientation are accurate at rates above chance; (4) as faces are perceived as more emotionally expressive, this evaluation shifts judgments of political orientation reliably to the political left. Overall, the results provide consistent empirical support for the primary theoretical claim: emotional expressivity helps explain how individuals can extract information about ideology from only facial cues.

Though persistent and robust to a variety of controls, the effect sizes we find are modest. Importantly, these analyses clearly imply that other facial traits besides emotional expressivity signal political orientation, and they just as clearly suggest that whatever these traits are, they go beyond broad social classifications such as age, gender, and ethnicity. There is some evidence these other traits may include the two dimension structure suggested by Todorov, ${ }^{82}$ though the findings are considerably stronger for trust than for dominance. Even though these studies suggest emotional expressivity clearly influences evaluations of political orientation and deserves further investigation, they also suggest that such political evaluations are extracted from a range of facial traits.

We should also note a set of caveats for these studies that are likely to be relevant for future research. Following the relevant literature, we used measures that treat ideology as a unidimensional left-right concept. While this approach is certainly defensible, there is a lively and ongoing debate about what ideology is (a coherent set of issue attitudes, a personality trait, a set of cognitive or information processing tendencies, or some combination of all of these?) and how it is best measured. ${ }^{83,84}$ Notably, there are persuasive arguments that ideology is a multidimensional concept, ${ }^{85}$ and it may be the case that not all of these dimensions map onto the same patterns as reported here. For example, it might be reasonable to hypothesize that the relationships we find are driven by social conservatism rather than economic conservatism, the underlying logic being that social conservatism is more likely to capture aspects of politics humans have dealt with for millennia, such as preferences on social hierarchy, adherence to traditional religious practices, and the treatment of rule breakers.

Similarly, while we employed multiple approaches to measuring emotional expressivity, these were primarily based on self-reported expressivity (Study 1) or perceived expressivity (Study 3 and 4). Only in Study 2 did we employ an objective measure of expressivity, and that on a single facial muscle. Other measurement approaches that more objectively capture a wider range of expressions are available and may be profitably employed in future research. Most notably, the Facial Action Coding System ${ }^{86}$ (FACS) has already been used by political scientists to effectively capture politically relevant nuances of facial emotional expression. ${ }^{87}$ FACS offers a validated objective approach to tapping emotional nuances and could be usefully employed to build upon the research reported here, especially when combined with more fine grained ideological measures.

Our findings also raise interesting questions on individual-level variation in preferences for facial expression. For example, do liberals prefer more facially expressive political leaders? Are any such political preferences for facial expressions context or culture dependent? Does facial physiology interact with, or influence perceptions of, emotional expressivity? Existing research provides ample basis for hypothesizing positive answers to both questions. ${ }^{88,89}$ Systematic investigation of these questions is beyond the scope of the present article but provides interesting directions for future research.

\section{References}

1. S. H. Friedman, T. I. Mertz, and M. R. DiMatteo, "Perceived bias in the facial expression of television news broadcasters," Journal of Communication, 1980, 30(4): 103-111.

2. G. Jahoda, "Political attitudes and judgements of other people," Journal of Abnormal and Social Psychology, 1954, 49: 331-334.

3. M. Koppensteiner and K. Grammer, "Motion patterns in political speech and their influence on personality ratings," Journal of Research in Personality, 2010, 44(3): 374-379.

4. L. Laustsen, "Decomposing the relationship between candidates' facial appearance and electoral success," Political Behavior, 2014, 36(4): 777-791.

5. G. J. McHugo and C. A. Smith, “The power of faces: A review of John T. Lanzetta's research on facial expression and emotion," Motivation \& Emotion, 1996, 2(2): 85-120.

6. C. Olivola and A. Todorov, "Elected in 100 milliseconds: Appearance-based trait inferences and voting," Journal of Nonverbal Behavior, 2010a, 34(2): 83-100.

7. C. Olivola and A. Todorov, "Fooled by first impressions? Reexamining the diagnostic value of appearance-based 


\section{Emotional expressivity as a predictor of ideology}

inferences," Journal of Experimental Psychology, 2010b, 46(2): 315-324.

8. C. Olivola, A. B. Sussman, K. Tsetsos, O. E. Kang, and A. Todorov, "Republicans prefer Republican-looking leaders: Political facial stereotypes predict candidate electoral success among right-leaning voters," Social Psychological and Personality Science, 2012, 3(5): 605-613.

9. D. G. Sullivan and R. D. Masters, “'Happy warriors': Leaders' facial displays, viewers' emotions, and political support," American Journal of Political Science, 1988, 32(2): 345-368.

10. P. Stewart and P. K. Dowe, "Interpreting President Barack Obama's facial displays of emotion: Revisiting the Dartmouth Group," Political Psychology, 2013, 34(3): 369-385.

11. A. Todorov, C. P. Said, A. D. Engel, and N. Oosterhof, "Understanding evaluation of faces on social dimensions," Trends in Cognitive Sciences, 2008, 12(12): 455-460.

12. N. Ambady, F. J. Bernieri, and J. A. Richeson, "Toward a histology of social behavior: Judgmental accuracy from thin slices of the behavioral system," in Advances in Social Psychology, M. P. Zanna, ed. (San Diego, CA: Academic Press, 2000), pp. 211-271.

13. Olivola and Todorov, 2010b.

14. K. Tskhay and N. Rule, "Accuracy in categorizing perceptually ambiguous groups: A review and meta-analysis," Personality and Social Psychology Review, 2013, 17(1): 72-86.

15. R. Bull and C. Hawkes, "Judging politicians by their faces," Political Studies, 1982, 30(1): 95-101.

16. C. M. Carpinella and K. L. Johnson, "Appearancebased politics: Sex-type facial cues communicate political party affiliation," Journal of Experimental Social Psychology, 2013, 49: 156-160.

17. Olivola and Todorov, 2010a.

18. Olivola and Todorov, 2010b.

19. T. Roberts, H. Griffin, P. W. McOwan, and A. Johnston, "Judging political affiliation from faces of UK MPs," Perception, 2011, 40(8): 949-952.

20. N. Rule and N. Ambady, "Democrats and Republicans can be differentiated from their faces," PLOS ONE, 2010, 5: e8733.

21. J. Samochowiec, M. Wanke, and K. Fiedler, "Political ideology at face value," Social Psychological and Personality Science, 2010, 1(3): 206-213.

22. M. Herrmann and S. Shikano, "Attractiveness and facial competence bias face-based inferences of candidate ideol-

ogy," Political Psychology, 2016, 37(3): 401-417, at p. 402.
23. Herrmann and Shikano, p. 414.

24. Samochowiec, Wanke, and Fiedler, 2010.

25. Olivola and Todorov, 2010b.

26. Carpinella and Johnson, 2013.

27. Olivola and Todorov, 2010b.

28. Samochowiec, Wanke, and Fiedler, 2010.

29. A. Todorov, C. Olivola, R. Dotsch, and P. MendeSidlecki, "Social attributions from faces: Determinants, consequences, accuracy, and functional significance," Annual Review of Psychology, 2015, 66: 519-545.

30. A. Todorov and B. Duchaine, "Reading trustworthiness in faces without recognizing faces," Cognitive Neuropsychology, 2008, 25(3): 1-16.

31. Todorov et al., 2008.

32. C. C. Ballew and A. Todorov, "Predicting political elections from rapid and unreflective face judgments," Proceedings of the National Academy of Sciences, 2008, 104(46): 17948-17953.

33. Laustsen, 2014.

34. P. Poutvaara, H. Jordahl, and N. Berggren, "Faces of politicians: Babyfacedness predicts inferred competence but not electoral success," Journal of Experimental Psychology, 2009, 45(5): 1132-1135.

35. G. Verhulst, M. Lodge, and H. Lavine, "The attractiveness halo: Why some candidates are perceived more favorably than others," Journal of Nonverbal Behavior, 2010, 34(2): 111-117.

36. Olivola et al., 2012.

37. Rule and Ambady, 2010.

38. C. Olivola, D. Tingley, and A. Todorov, The Donkey in Elephant's Clothing: Revising the Impact and Validity of Political Facial Stereotypes (Pittsburgh, PA: Tepper, School of Business, Carnegie Mellon University, 2014).

39. Todorov et al., 2015, p. 532.

40. Olivola and Todorov, 2010b, p. 323.

41. Samochoweic, Wanke, and Fiedler, 2010, p. 211.

42. K. L. Schmidt and J. F. Cohn, "Human facial expressions as adaptations: Evolutionary questions in facial expression research," American Journal of Physical Anthropology, 2001, Suppl. 33: 3-24.

43. P. Ekman, "Facial expressions," in The Handbook of Cognition and Emotion, T. Dalgleish and M. Power, eds. (New York: Wiley, 1999), pp. 301-320.

44. J. Cohn, K. Schmidt, R. Gross, and P. Ekman, "Individual differences in facial expression: Stability over time, 


\section{Peterson et al.}

relation to self-reported emotion, and ability to inform person identification," in paper presented at the 4th IEEE International Conference on Multimodal Interfaces (Pittsburgh, PA: 2002).

45. Schmidt and Cohn, 2001, p. 17.

46. M. Van Vugt and G. E. Allen, "The many faces of leadership," Current Directions in Psychological Science, 2015, 6: 484-489.

47. L. Laustsen and M. B. Petersen, "Does a competent leader make a good friend? Conflict, ideology and the psychologies of friendship and followership" Evolution and Human Behavior, 2015, 36(4): 286-293.

48. Todorov et al., 2015.

49. A. M. Kring and A. H. Gordon, "Sex differences in emotion: expression, experience and physiology," Journal of Personality and Social Psychology, 1998, 74(3): 686-703.

50. S. Hareli, N. Shomrat, and U. Hess, "Emotional versus neutral expressions and perceptions of social dominance and submissiveness," Emotion, 2009, 9(3): 378-384.

51. J. J. Gross and J. P. Oliver, "Facets of emotional expressivity: Three self-report factors and their correlates," Personality and Individual Differences, 1995, 19(4): 555-568.

52. J. J. Gross and J. P. Oliver, "Revealing feelings: Facets of emotional expressivity in self-reports, peer ratings and behavior," Journal of Personality and Social Psychology, 1998, 72(2): 435-448.

53. J. J. Gross and J. P. Oliver, "Individual differences in two emotion regulation processes: implications for affect, relationships and well-being," Journal of Personality and Social Psychology, 2003, 85(2): 348-362.

54. L. Leone and A. Chirumbolo, "Conservatism as motivated avoidance of affect: Need for affect scales predict conservatism measures," Journal of Research in Personality, 2008, 42(3): 755-762.

55. E. Constantini and K. H. Craik, "Personality and politicians: California party leaders, 1960-1976," Journal of Personality and Social Psychology, 1980, 38(4): 641-661.

56. R. Pliskin, D. Bar-Tal, G. Sheppes, and E. Halperin, "Are leftists more emotion-driven than rightests? The interactive influence of ideology and emotions on support for policies," Personality and Social Psychology Bulletin, 2014, 40(12): 1681-1697.

57. Gross and Oliver, 1995.

58. J. R. Hibbing, K. B. Smith, and J. R. Alford, Predisposed (New York: Routledge, 2013).

59. Gross and Oliver, 1995.

60. Gross and Oliver, 1998.

61. Gross and Oliver, 1995.
62. Gross and Oliver, 1998.

63. Gross and Oliver, 1995.

64. J. L. Napier and J. T. Jost, "Why are conservatives happier than liberals?" Psychological Science, 2008, 19(6): $565-572$.

65. B. R. Schenckler, J. R. Chambers, and B. M. Le, "Conservatives are happier than liberals, but why? Political ideology, personality, and life satisfaction," Journal of Research in Personality, 2012, 46(2): 127-146.

66. Gross and Oliver, 1995 , p. 558.

67. F. Newport, "American's church attendance inches up in 2010," Gallup, June 25, 2010, http:/www.gallup.com/p oll/141044/americans-church-attendance-inches-2010.aspx (accessed November 28, 2017).

68. J. T. Larson, C. J. Norris, and J. T. Cacioppo, "Effects of positive and negative affect on electromyographic activity over zygomaticus major and corrugator supercilii," Psychophysiology, 2003, 40(5): 776-785.

69. J. R. Hibbing, K. B. Smith, and J. R. Alford, "Differences in negativity bias underlie variations in political ideology," Behavioral and Brain Sciences, 2014, 37(3): 297-350.

70. J. J. Gross, “Antecedent- and response-focused emotion regulation: Divergent consequences for experience, expression, and physiology," Journal of Personality and Social Psychology, 1998, 74(1): 224-237.

71. J. J. Gross and R. Levenson, "Emotional suppression: Physiology, self-report, and expressive behavior," Journal of Personality and Social Psychology, 1993, 64(6): 970-986.

72. M. K. Greenwald, E. W. Cook, and P. J. Lang, "Affective judgment and psychophysiological response: Dimensional covariation in the evaluation of pictorial stimuli," Journal of Pyschophysiology, 1989, 3(1): 51-64.

73. S. D. Kreibig, F. H. Wilhelm, W. T. Roth, and J. Gross, "Cardiovascular, electrodermal and respiratory response patterns to fear- and sadness-inducing films," Psychophysiology, 2017, 44(5): 787-806.

74. L. G. Tassinary, J. T. Caccioppo, and E. Vanman, "The skeletomotor system: Surface electromyography," in Handbook of Psychophysiology, 3rd ed. J. T. Caccioppo, L. G.

Tassinary, and G. G. Berntson, eds. (New York: Cambridge University Press, 2007), pp. 267-299.

75. P. J. Lang, M. M. Bradley, and B. N. Cuthbert, "International affective picture systems (IAPS): Affective ratings of pictures and instruction manual," Technical Report A-6, 2005, University of Florida, Gainesville, FL.

76. Larson, Norris, and Cacioppo, 2003

77. S. Hix, A. Noury, and G. Roland, "Roll call votes in the European Parliament. July 26,” 2011, http://personal.lse.ac .uk/hix/HixNouryRolandEPdata.HTM (accessed September $15,2014)$. 


\section{Emotional expressivity as a predictor of ideology}

78. For a survey, see Todorov et al., 2015.

79. N. Oosterhof and A. Todorov, "The functional basis of face evaluation," Proceedings of the National Academy of Sciences, 2009, 105: 11087-11092.

80. Oosterhof and A. Todoro, 2009.

81. Gross and Oliver, 1995.

82. Todorov et al., 2015.

83. J. Gerring, "Ideology: A definitional analysis," Political Research Quarterly, 1997, 50(4): 957-994.

84. J. T. Jost, C. M. Federico, and J. L. Napier, "Political ideology: Its structure, functions and elective affinities," Annual Review of Psychology, 2009, 60: 307-337.
85. S. Feldman and C. Johnston, "Understanding the determinants of political ideology: Implications of structural complexity," Political Psychology, 2014, 35(3): 337-358.

86. P. Ekman and V. W. Friesen, "Felt, false, and miserable smiles," Journal of Nonverbal Behavior, 1982, 6(4): 238-252.

87. Stewart and Dowe, 2013.

88. P. Stewart, B. Waller, and J. Schubert, "Presidential speechmaking style: Emotional response to microexpressions of facial affect," Motivation and Emotion, 2009, 33(2): 125-135.

89. Laustsen and Petersen, 2015. 